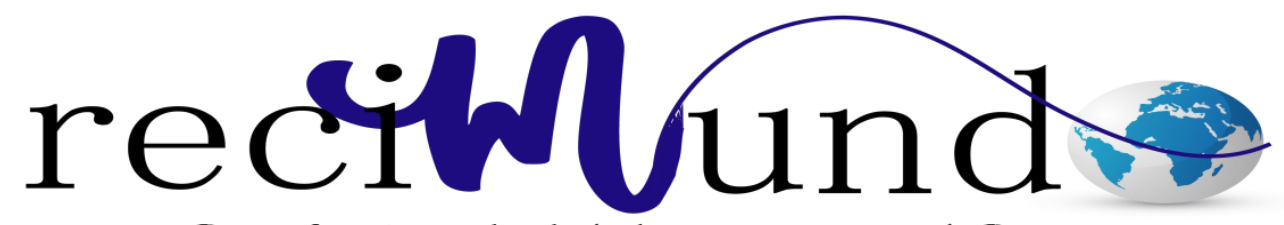

Revista Científica Mundo de la Investígación y el Conocimiento

Anita María Murillo Zavala ${ }^{\text {a }}$; Elsa Noralma Lucas Parrales ${ }^{\text {b }}$; Javier Martín Reyes

Baque $^{c}$; Zulbey Rivero de Rodríguez ${ }^{\mathrm{d}}$

Parasitosis intestinal asociado a factores epidemiológicos en pacientes pediátricos

Revista Científica Mundo de la Investigación y el Conocimiento. Vol. 1 núm., 5, diciembre, 2017, pp. 846-859

DOI: $10.26820 /$ recimundo/1.5.2017.846-859

Editorial Saberes del Conocimiento

a. Universidad Estatal del Sur de Manabí; anita.murillo@unesum.edu.ec

b. Universidad Estatal del Sur de Manabí; elsa.lucas@unesum.edu.ec

c. Universidad Estatal del Sur de Manabí; javier.reyes@unesum.edu.ec

d. Universidad Estatal del Sur de Manabí; zulbey.rivero@unesum.edu.ec 


\section{Parasitosis intestinal asociado a factores epidemiológicos en pacientes pediátricos}

Vol. 1, núm. 5., (2017)

Anita María Murillo Zavala; Elsa Noralma Lucas Parrales; Javier Martín Reyes Baque; Zulbey Rivero de Rodríguez

\section{RESUMEN}

El estudio realizado en el recinto Las Piñas, tuvo como objetivo principal: Determinar la parasitosis intestinal asociado a factores epidemiológicos en pacientes pediátricos del recinto Las Piñas de Julcuy. Se aplicaron estudios no experimental, analítico y transversal durante el periodo Mayo-Septiembre 2015. Se aplicaron técnica de encuesta, ficha de Laboratorio, ficha de resultados y técnicas de laboratorio para análisis de muestras fecales como microscopia directa y sedimentación espontanea en tubo, para determinar presencia de parásitos presente en los pacientes fueron analizadas 180 muestras de niños en edades 1 a 14 años. En la determinación de los factores epidemiológico se realizó una encuesta a las madres de familia con relación a cada paciente. Donde se obtuvo los siguientes resultados: $89 \%$ de madres desconocen cómo se transmiten los parásitos, $85 \%$ realiza la eliminación de sus excretas en pozos sépticos, $77 \%$ habita en casa de caña, $67 \%$ de niños lava en ocasiones los alimentos antes de consumirlos y $65 \%$ de la población consume agua de pozo. La prevalencia de parasitosis mediante la técnica de microscopia directa y la sedimentación espontanea en tuvo, fue Entamoeba histolytica 36\%, Giardia lamblia 21\%, Entamoeba coli 12\%, Blastocystis hominis 12\%, Áscari lumbricoides 7\%, Hymenolepis nana 6\%, Trichiura trichuris 3\%, y Enterobius vermicularis 3\%. La edad de prevalencia de parasitosis fue de 10 a 14 años afectando la parasitosis a ambos sexos.

Palabras claves: Parasitosis intestinal; factores epidemiológicos, prevalencia, pacientes pediátricos. 


\section{Parasitosis intestinal asociado a factores epidemiológicos en pacientes pediátricos}

Vol. 1, núm. 5., (2017)

Anita María Murillo Zavala; Elsa Noralma Lucas Parrales; Javier Martín Reyes Baque; Zulbey Rivero de Rodríguez

ABSTRACT

The reasch carried out in Las Piñas, town it had as main objective: To determine the intestinal parasitosis associated factors epidemologics in pediatric patients of Las piñas town Julcuy we applied not experimental, analytical and transversal studies during the period from May to September 2015, we applied record Laboratory, record results and laboratory techniques for analysis of fecal samples as direct microscopy and sedimentation tube to determine the presence of parasites in patients. Also we analyzed 180 samples of in children aged 1 to 14 years. For determining the epidemiological factors a survey was carried out of mothers in relation to each patient was performed. Where the following results were obtained: $89 \%$ of mothers unknown how parasites are transmitted, $85 \%$ performed the elimination their septicwen, $77 \%$ live at home cane, $67 \%$ of children occasionally wash food before eating and $65 \%$ of the population consume well water. The prevalence of parasitism by the technique of direct microscopy and sedimentation had was 36\% Entamoeba histolytica, Giardia lamblia 21\%, $12 \%$ coli Entamoeba, Blastocystis hominis 12\%, 7\% Ascari lumbricoides, Hymenolepis nana 6\%, Trichura trichuris 3 $\%$, and 3\% Enterobius vermicularis. The prevalence of parasitosis age was 10 to 14 years parasitosis affecting both sexes.

Key words: Intestinal parasitosis; epidemiological, prevalence, pediatric patients. 


\section{Parasitosis intestinal asociado a factores epidemiológicos en pacientes pediátricos}

Vol. 1, núm. 5., (2017)

Anita María Murillo Zavala; Elsa Noralma Lucas Parrales; Javier Martín Reyes Baque; Zulbey

Rivero de Rodríguez

\section{Introducción.}

"La parasitosis intestinal a nivel mundial y en Latinoamérica se han considerado según estudios y diagnósticos médicos, como infecciones producidas por parásitos cuyo hábitat natural es el aparato digestivo de todo ser humano". ${ }^{1-3}$

“En el Ecuador hay 3’643.806 niños y niñas menores de doce años que se encuentran en condiciones no saludables y con enfermedades intestinales, una de las diez causas principales de consulta pediátrica en los servicios de Salud de todo el país". ${ }^{4-6}$

En Manabí, el 98\% de casos de parasitosis intestinal se da por la prevalencia de características climáticas, condiciones de insalubridad y pobreza. Se han relacionado a diversos estudios a nivel nacional y en la provincia se refieren altas incidencias de infección.

En la Parroquia Julcuy perteneciente al Cantón Jipijapa se presentaron hasta mediados del año un total de 455 pacientes con sintomatología parasitaria, que encasillan a pacientes pediátricos registros proporcionado por el Centro de Salud Rural Julcuy, siendo más afectado el recinto Las Piñas, por ente se realizó un estudio a los niños en edades comprendidas de 1 - 14 años con el tema parasitosis intestinal asociado a factores epidemiológicos en pacientes pediátricos del recinto las Piñas de Julcuy a tal punto de determinar los parásitos presentes y los factores que los producen.

Se aplicó un estudio No experimental, Analítico y Transversal, mediante la aplicación de técnicas como encuesta, ficha de laboratorio y ficha de resultados. Utilizando métodos de Laboratorio como el examen parasitológico directo y la sedimentación en tubo. Los resultados de 


\section{Parasitosis intestinal asociado a factores epidemiológicos en pacientes pediátricos}

Vol. 1, núm. 5., (2017)

Anita María Murillo Zavala; Elsa Noralma Lucas Parrales; Javier Martín Reyes Baque; Zulbey Rivero de Rodríguez

las muestras procesadas fueron analizados para la recolección de datos a fin de dar respuesta positiva al problema e hipótesis planteadas.

La presente investigación tuvo como objetivo determinar parasitosis intestinal asociados a factores epidemiológicos en pacientes pediátricos del recinto las piñas de Julcuy.

\section{Metodología.}

El estudio se realizó en el recinto Las Piñas de la parroquia Julcuy perteneciente al Cantón Jipijapa localizada en la zona sur con una población de 450 habitantes, los pacientes son niños en edad comprendida de 1 - 14 años de esta comunidad. Para este estudio se aplicó las normas de bioética además el consentimiento informado por parte de las madres de familia para poder ser procesada las muestras.

Previo a esto se les brindó una charla introductora dirigido a los padres y madres de familia sobre que son los parásitos, como afectan y las complicaciones que pueden provocar estos, también se les explico la debida recomendación de cómo deben recoger cada una de las muestras, evitar la contaminación ya sea esta con la orina o con cualquier otra sustancia.

Para esto se les entregó un recipiente estéril boca ancha con tapa de rosca, en donde deben introducir la muestra, tiene que ser al tamaño de una nuez 3 a $5 \mathrm{gr}$ y si esta es líquida colocar una porción mayor y se fijó una fecha indicada para la recolección de las muestras por parte del investigador para el debido análisis. 


\section{Parasitosis intestinal asociado a factores epidemiológicos en pacientes pediátricos}

Vol. 1, núm. 5., (2017)

Anita María Murillo Zavala; Elsa Noralma Lucas Parrales; Javier Martín Reyes Baque; Zulbey Rivero de Rodríguez

Una vez recolectada las muestras fecales fueron identificadas con códigos de acuerdo a cada paciente y fueron trasladadas al laboratorio aplicando las normas de bioseguridad y la cadena de conservación de espécimen, estás no excedieron de 2 a 4 horas durante su transporte.

\section{Técnica de examen microscópico directo}

Como primer paso, las muestras fueron analizadas identificando su color, olor y consistencia luego se procedió a colocar en una lámina portaobjeto una gota de solución salina o lugol, luego se colocó aproximadamente $2 \mathrm{~g}$ de heces.

Se hiso un homogenizado encima de la lámina con un palillo, hasta obtener una muestra diluida y se colocó una laminilla cubre objeto finalmente se observó al microscopio enfocando con el lente de 10X y 40X y se procedió a reportar la existencia de parásitos por cada campo.

\section{Técnica de sedimentación en tubo}

Esta técnica se basó en separar aproximadamente $4 \mathrm{~g}$ de heces de cada muestra de los pacientes y se hiso un homogenizado en $10 \mathrm{ml}$ de solución salina hasta que se logró una suspensión adecuada.

La mezcla se vertió en un tubo cónico de plástico o vidrio de 10ml, filtrándola a través de gasa. Luego se completó el volumen del tubo con solución salina y se tapó herméticamente. Se procedió a agitar por 30 segundos y se dejó reposar por 45 minutos.

Se eliminó el sobrenadante y con una pipeta se tomó una muestra del fondo del tubo se coloca 1 gota en una placa porta objeto, agregándole una gota de lugol o solución salina. 


\section{Parasitosis intestinal asociado a factores epidemiológicos en pacientes pediátricos}

Vol. 1, núm. 5., (2017)

Anita María Murillo Zavala; Elsa Noralma Lucas Parrales; Javier Martín Reyes Baque; Zulbey Rivero de Rodríguez

Finalmente las láminas portaobjetos se cubrieron con laminillas cubre objeto y se observó al microscopio con lente de 10X Y 40X reportando la existencia de parásitos por cada campo.

Con el apoyo del distrito de salud y con el consentimiento informado por parte de los padres, los resultados se manejaron con código y cumplimiento de las normas de Bioética. Para el análisis y presentación de los resultados se utilizaron la estadística descriptiva y se utilizaron las herramientas de paquetes de datos SPSS.

\section{Resultados.}

Obtenidos los resultados de la investigación mediante la encuesta dirigida a las madres de familia y las técnicas de laboratorio empleadas para el análisis de las muestras fecales se determinó los siguientes resultados de acuerdo a cada pregunta y análisis realizado en el análisis microscópico.

Que el $89 \%$ de las madres de familia desconocen cómo se contraen los parásitos, mientras que un $11 \%$ si tienen conocimiento de cómo se contraen, del total del $100 \%$ de las encuestas realizadas.

Del $100 \%$ de las encuestas realizadas a las madres, se dedujo que el $65 \%$ consume agua de pozo, el $35 \%$ consume agua de rio y mientras que el $16 \%$ consume agua entubada.

Dentro de una de las preguntas planteadas a las madres de familia en relación a cada muestra entregada de cada paciente dio como resultado: que el $62 \%$ a veces realiza el lavado de manos antes de consumir los alimentos, el $31 \%$ si realiza el lavado de manos, mientras que el $7 \%$ no lo realiza, del $100 \%$ de la encuesta realizada. 


\section{Parasitosis intestinal asociado a factores epidemiológicos en pacientes pediátricos}

Vol. 1, núm. 5., (2017)

Anita María Murillo Zavala; Elsa Noralma Lucas Parrales; Javier Martín Reyes Baque; Zulbey Rivero de Rodríguez

En relación con la muestra entregada, si el niño o niña lava los alimentos antes de consumirlos las madres respondieron en la encuesta realizada del 100\%: que el 67\% a veces lo realiza, el 28\% no lo realiza y el $5 \%$ si realiza el lavado de los alimentos antes de consumirlos.

Una de las preguntas formulada fue si el niño o niña tenía contacto con algún tipo de animal doméstico dando como resultado del 100\% de las encuesta realizadas que: el $34 \%$ tiene contacto con perro, el $23 \%$ no tiene contacto con ningún animal, el $17 \%$ tiene contacto con chanchos, el $16 \%$ con chivo y un $10 \%$ con gato.

Del $100 \%$ de la encuesta realizada sobre el tipo de vivienda dio como resultado: que el $77 \%$ habita en casa de caña, un $12 \%$ en estructura de hormigón, mientras que un $11 \%$ en estructura mixta.

Unas de las preguntas formulada fue si el niño o niña había presentado signos y síntomas lo cual respondieron que el: 25\% no había presentado ningún síntoma, el 17\% había presentado diarrea, el 14\% picazón anal, el 13\% falta de apetito, el 11\% dolor abdominal, el 6\% pérdida de peso y un 5\% fiebre del $100 \%$ de la encuesta realizada.

Dentro de la disposición para la eliminación de excretas que ellos utilizan en sus hogares las madres de familia respondieron: que el $86 \%$ utiliza pozo séptico, el $8 \%$ lo realiza de forma inadecuada al aire libre y el $7 \%$ consta con letrina en sus hogares del $100 \%$ de la encuesta realizada. 


\section{Parasitosis intestinal asociado a factores epidemiológicos en pacientes pediátricos}

Vol. 1, núm. 5., (2017)

Anita María Murillo Zavala; Elsa Noralma Lucas Parrales; Javier Martín Reyes Baque; Zulbey Rivero de Rodríguez

Del $100 \%$ de la encuesta realizada a las madres de familia respondieron en cuanto a la presencia de vectores dentro de su hogar: el $48 \%$ de presencia de mosca, $22 \%$ presencia de ratón, $19 \%$ de mosquito, $6 \%$ de cucarachas, mientras que el $5 \%$ no cuenta con ningún tipo de vectores en sus hogares.

En relación a los niños y niñas en la encuesta formulada a las madres el $51 \%$ de ellos camina descalzo, mientras que el $49 \%$ no lo realiza del 100\% de las encuestas realizadas.

En relación a los niños y niñas si juegan con tierra las madres contestaron del 100\% de la encuesta realizada que el $57 \%$ no juega con tierra, mientras que un $43 \%$ si lo realiza.

Como conclusión del objetivo de analizar los posibles factores de riesgo asociados a la parasitosis intestinal se determinó que los factores que afectan a la población del recinto piñas en su mayor porcentaje $89 \%$ es la falta de conocimiento sobre que es la parasitosis, lo que ocasionan que ellos no lleven una higiene adecuada para así controlar la parasitosis. Mientras que los habitantes en un $85 \%$ utilizan para la eliminación de excretas pozos sépticos inadecuado, ya que un $8 \%$ de ellos, en ocasiones lo realizan al aire libre (inadecuado), produciendo un riesgo latente en el suelo. Debido al factor socioeconómico que afecta al recinto piñas, el $77 \%$ de los habitantes habita en casa de caña gran parte de ellas se encuentran en el suelo siendo fácil la adquisición de parásitos para los niños del hogar, ya que en un 67\% de ellos no realiza el lavado de alimentos antes de consumir estos. $\mathrm{Y}$ un factor importante que provoca el contagio de parásitos es el consumo de agua de pozo que ellos utilizan que corresponde a un $65 \%$, lo cual tiene un tratamiento adecuado paras su consumo. 


\section{Parasitosis intestinal asociado a factores epidemiológicos en pacientes pediátricos}

Vol. 1, núm. 5., (2017)

Anita María Murillo Zavala; Elsa Noralma Lucas Parrales; Javier Martín Reyes Baque; Zulbey Rivero de Rodríguez

En el análisis microscópico realizado a cada una de las muestras dentro del laboratorio y aplicando cada una de las normas de bioética, arrojaron los siguientes resultados de un total de 180 muestras analizadas: la presencia de parásitos como:

- Entamoeba histolytica un $74 \%$ de pacientes pediátricos, si poseen este parasito mientas que un $26 \%$ no posee este parasito del $100 \%$ de muestras analizadas.

- Giardia lamblia: un 57\% de pacientes pediátricos, si poseen mientras que un $43 \%$ no poseen este tipo de parasito del $100 \%$ de muestras analizadas.

- Entamoeba coli un $25 \%$ de pacientes pediátricos si posee este parasito mientras que un $75 \%$ no posee este parasito del $100 \%$ de las muestras analizadas.

- Blastocystis hominis, un $24 \%$ de los pacientes pediátricos en estudio poseen este parasito mientras que un $76 \%$ no poseen, del $100 \%$ de muestras analizadas.

- Áscari lumbricoides, un $15 \%$ de pacientes pediátricos si poseen este parásito, mientras que un $85 \%$ no poseen este parásitos en su organismo del $100 \%$ de muestra analizadas.

- Hymenolepis nana, un $12 \%$ de pacientes pediátricos si poseen este parasito, mientras que un $88 \%$ de ellos no lo posee en su organismo del $100 \%$ de muestras analizadas.

- Trichiura trichuris, un 7\% de pacientes pediátricos si poseen este parasito, mientras que un $93 \%$ no posee en su organismo del 100\% de muestras analizadas.

- Enterobius vermicularis, un $7 \%$ de pacientes pediátricos si posee este parasito mientras que el $93 \%$ no lo posee en su organismo del 100\% de muestras analizadas. 


\section{Parasitosis intestinal asociado a factores epidemiológicos en pacientes pediátricos}

Vol. 1, núm. 5., (2017)

Anita María Murillo Zavala; Elsa Noralma Lucas Parrales; Javier Martín Reyes Baque; Zulbey Rivero de Rodríguez

Como conclusión del objetivo de Identificar parásitos intestinales, en materia fecales

mediante las técnicas microscópicas y sedimentación espontanea en tubo, en pacientes pediátricos del recinto las Piñas de Julcuy dio como resultado del total de 180 muestras analizadas, la presencia de parásitos los cuales fueron reportados. Donde prevaleció la Entamoeba histolytica en un $36 \%$, seguido del parasito Giardia lamblia $21 \%$, Entamoeba coli 12\%, Blastocystis hominis 12\%, áscaris lumbricoides 7\%, Hymenolepis nana 6\%, Trichiura trichuris $3 \%$ y Enterobius vermicularis 3\% del 100\% de la muestras analizadas afectando a ambos sexos y la edad que predomino la parasitosis fue de 10 a 14 años.

En relación a los factores epidemiológicos con la prevalencia de parásitos intestinales dio como resultado que el desconocimiento de cómo se contraen los parasito influye en la infección por Entamoeba histolytica con la frecuencia 119 casos, 67 por Giardia lamblia y 39 por Entamoeba coli; 38 Blastocystis hominis; 24 áscaris lumbricoides; 21 Hymenolepis nana; 11 Enterobius vermicularis y Trichiura trichuris.

Se puedo evidenciar que en relación a la eliminación de excretas con los parásitos el pozo séptico influye con un frecuencia de 114; con la frecuencia de 74 Giardia lamblia; 38 de frecuencia Entamoeba coli; Blastocystis hominis con una frecuencia de 37; Áscari lumbricoides con una frecuencia de 23 y Hymenolepis nana presento una frecuencia de 17. La de mayor frecuencia fue Entamoeba Histolytica relacionada a la eliminación de excreta en pozos sépticos.

Se observó que en relación al consumo de agua de pozo existe un predomino de Entamoeba histolytica con una frecuencia de 81; 57 de Giardia lamblia; 29 Entamoeba coli; 27 Blastocystis hominis; 20 Áscari lumbricoides; 12 Hymenolepis nana; trichuris 6; Enterobius 


\section{Parasitosis intestinal asociado a factores epidemiológicos en pacientes pediátricos}

Vol. 1, núm. 5., (2017)

Anita María Murillo Zavala; Elsa Noralma Lucas Parrales; Javier Martín Reyes Baque; Zulbey Rivero de Rodríguez

vermicularis 5; en relación al relación al consumo de agua de rio los resultados no son significativos.

De acuerdo al tipo de vivienda se presentó una incidencia de Entamoeba histolytica 99; Giardia lamblia 65; Blastocystis hominis y Entamoeba coli con frecuencia de 35; Hymenolepis nana 16; Áscari lumbricoides 22; Trichiura trichuris 7 y Enterobius vermicularis 9. Observándose en mayor frecuencia en relación al tipo de vivienda de caña relacionada a la parasitosis.

Al relacionar la higiene con la parasitosis se evidencio que predomino 88 de Entamoeba histolytica; 42 de Giardia lamblia; 30 Blastocystis hominis; 23 Entamoeba coli; 20 Áscari lumbricoides; 12 Hymenolepis nana y Enterobius vermicularis 9. Observándose en mayor frecuencia al lavado de manos solo a veces.

Concluido los resultados tanto de las encuesta realizada a las madres de familia, como la de las prueba de laboratorio empleada a cada una de las muestras fecales y observar un alto índice de parasito y factores epidemiológico que contribuyen a esta parasitosis. Como investigador se procede a plantear una propuesta para futuras investigaciones con el tema: Capacitación sobre parasitosis intestinal dirigido a madres de familia del recinto las piñas en conjunto con el centro de salud rural - Julcuy. Por medio de este tipo de propuesta se busca mejorar la calidad de vida de los niños disminuyendo la parasitosis y controlando los factores epidemiológicos que la provocan la parasitosis. 


\section{Parasitosis intestinal asociado a factores epidemiológicos en pacientes pediátricos}

Vol. 1, núm. 5., (2017)

Anita María Murillo Zavala; Elsa Noralma Lucas Parrales; Javier Martín Reyes Baque; Zulbey Rivero de Rodríguez

\section{Conclusiones.}

Al término de la investigación se pudo concluir que:

El principal factor epidemiológico que afecta a la población es el desconocimiento de información sobre la parasitosis, lo que conduce a ellos que consuman alimentos inadecuados y no apliquen higienes correctas en sus hogares, ya que nunca han tenido charlas o intervenciones en su población sobre la investigación tratada.

Mediante el análisis coprológico realizado a los pacientes pediátricos de 1 a 14. Se pudo determinar el alto índice de parásitos como Entamoeba Histolytica, Giardia lamblia, Áscari lumbricoides, trichuris Trichiura., Entamoeba colli. Hymenolepis nana, Blastocystis hominis y Enterobius vermicularis, debido a las malas higienes aplicadas en sus hogares, por el total desconocimiento sobre la parasitosis.

La muestra lo conformaron 180 pacientes pediátricos, donde 101 fueron de sexo masculino y 79 pacientes del sexo femenino, los rangos analizados fueron 1-4,5-9 y 10-14, dando como resultado que hubo más prevalencia en la edad de 10-14 años. Lo cual ellos desconocen cómo se contraen los parásitos, realizan su eliminación de excretas en pozos sépticos, no aplican una higiene adecuada, viven en casas de caña y consumen agua de pozo inadecuada.

En la hipótesis planteada se concluye que la población en estudio si presento parásitos, ya que uno de los factores que conduce a esta parasitosis es el consumo de agua de pozo que no tiene un control de calidad y las malas higienes por parte de los pacientes pediátricos. 


\section{Parasitosis intestinal asociado a factores epidemiológicos en pacientes pediátricos}

Vol. 1, núm. 5., (2017)

Anita María Murillo Zavala; Elsa Noralma Lucas Parrales; Javier Martín Reyes Baque; Zulbey

Rivero de Rodríguez

\section{Bibliografía.}

1. Espinosa Morales M, Alazales Javiqué M, García Socarrás AM. Parasitosis intestinal, su relación con factores ambientales en niños del sector "Altos de Milagro", Maracaibo. Revista Cubana de Medicina General Integral. 2011; 27(3): p. 396-405.

2. Castillo Núñez B, Iribar Moreno M, Segura Prevost R, Salvador Álvarez M. Prevalencia de parasitismo intestinal en la población infantil perteneciente al policlínico "4 de agosto" de Guantánamo. Medisan. 2002 Feb; 6(1): p. 46-52.

3. Cárdenas M, Martínez R. Protozoarios parásitos de importancia en salud pública. Rev. Perubiol. 2004 Jul; 11(2): p. 149-152.

4. Pérez Sánchez G, Redondo de la Fé G, Fong Rodríguez HG, Sacerio Cruz M. Prevalencia del parasitismo intestinal en los niños. Medisan. 2014 Abr; 16(4): p. 551-557.

5. Brooks C, Butel J, Morse S. Microbiología Médica. 16th ed. México: El Manual Moderno; 1999.

6. Oberhelman R, Guerrero E, Fernández M, Silio M. Correlation between intestinal parasitosis, physical growth and psychomotor development among infants and children from rural Nicaragua. Am J Trop Med Hyg. 1998 Jua; 58(4): p. 470-475. 